Article

\title{
A Modified Differential Coherent Bit Synchronization Algorithm for BeiDou Weak Signals with Large Frequency Deviation
}

\author{
Zhifeng Han * $\mathbb{D}$, Jianye Liu, Rongbing Li, Qinghua Zeng and Yi Wang \\ College of Automation Engineering, Nanjing University of Aeronautics and Astronautics, Nanjing 211100, \\ China; ljyac@nuaa.edu.cn (J.L.); lrbing@nuaa.edu.cn (R.L.); zengqh@nuaa.edu.cn (Q.Z.); \\ wangyinuaanrc@163.com (Y.W.) \\ * Correspondence: hanzhifeng@nuaa.edu.cn; Tel.: +86-025-8489-2304
}

Received: 18 May 2017; Accepted: 1 July 2017; Published: 4 July 2017

\begin{abstract}
BeiDou system navigation messages are modulated with a secondary $\mathrm{NH}$ (Neumann-Hoffman) code of $1 \mathrm{kbps}$, where frequent bit transitions limit the coherent integration time to 1 millisecond. Therefore, a bit synchronization algorithm is necessary to obtain bit edges and $\mathrm{NH}$ code phases. In order to realize bit synchronization for BeiDou weak signals with large frequency deviation, a bit synchronization algorithm based on differential coherent and maximum likelihood is proposed. Firstly, a differential coherent approach is used to remove the effect of frequency deviation, and the differential delay time is set to be a multiple of bit cycle to remove the influence of $\mathrm{NH}$ code. Secondly, the maximum likelihood function detection is used to improve the detection probability of weak signals. Finally, Monte Carlo simulations are conducted to analyze the detection performance of the proposed algorithm compared with a traditional algorithm under the CNOs of $20 \sim 40 \mathrm{~dB}-\mathrm{Hz}$ and different frequency deviations. The results show that the proposed algorithm outperforms the traditional method with a frequency deviation of $50 \mathrm{~Hz}$. This algorithm can remove the effect of BeiDou NH code effectively and weaken the influence of frequency deviation. To confirm the feasibility of the proposed algorithm, real data tests are conducted. The proposed algorithm is suitable for BeiDou weak signal bit synchronization with large frequency deviation.
\end{abstract}

Keywords: bit synchronization; NH code; differential coherent; Beidou System

\section{Introduction}

With the development of Global Navigation Satellite System (GNSS), high-sensitivity receiver design in weak signal environments such as urban canyons, tunnels and dense foliage has become an important issue [1-7]. In high-sensitivity receivers, the extension of integration time is a typical strategy to enhance the signal-to-noise ratio (SNR) [8]. However, such extension is limited by data bit reversions [9].

The BeiDou Navigation Satellite System is called BeiDou System for short and is scheduled to be completed in 2020. A secondary modulation of the Neumann-Hoffman $(\mathrm{NH})$ code is adopted by the BeiDou System, which plays an important role in spectral separation and narrowband interference protection [10]. However, the periodicity of the transmitted sequence is modified by the NH codes, leading to more polarity changes [11,12]. The data bit rate increases to $1 \mathrm{kbps}$ and the integration time is limited to 1 millisecond.

In the Beidou System, once signal acquisition phase is completed, a bit synchronization phase should be used to detect the position of the data bit edges and $\mathrm{NH}$ codes in order to extend the coherent integration time of the tracking loops [13]. 
The classic algorithm for GPS data bit synchronization is the histogram method. It labels the sign changes in consecutive correlation results [14]. If the sign change occurs, the corresponding counter would increase. When the largest counter value exceeds the threshold, the bit boundary is found. Because of its simplicity, the histogram method has been widely used in consumer-level receivers, but it is only adequate for carrier-to-noise ratio $(\mathrm{C} / \mathrm{N} 0)$ above $30 \mathrm{~dB}-\mathrm{Hz}$, which is the normal condition for most outdoor GNSS receivers. When the $\mathrm{C} / \mathrm{N} 0$ is less than $30 \mathrm{~dB}-\mathrm{Hz}$, the performance slides fast.

The Viterbi algorithm is also used for bit synchronization [15]. It is adequate for C/N0 even of less than $20 \mathrm{~dB}-\mathrm{Hz}$, but its disadvantage is the complicated calculation and increased complexity. For GPS weak signals, the maximum likelihood (ML) bit synchronization method is more popular [16,17]. The ML method calculates the bit energy of all possible navigation bit boundaries. The peak of bit energies corresponds to the bit boundary. This method still has good performance when $\mathrm{C} / \mathrm{N0}$ is $20 \mathrm{~dB}-\mathrm{Hz}$, but the ML method is sensitive to the frequency deviation. If the frequency deviation is larger than $25 \mathrm{~Hz}$, the ML method is nullified [16,18]. Furthermore, under low C/N0, the frequency deviation is unavoidable and the ML is unreliable.

An efficient differential coherent accumulation algorithm (EDCAA) for weak GPS signal bit synchronization in [19] can eliminate the influence of frequency deviation by adopting the differential coherent value of two consecutive correlation outputs. Authors in [20] proposed a balance differential coherent bit synchronization algorithm for GPS signals, which adopts two complementary differential distances to avoid the unbalanced problem and the accumulation attenuation. Because these two algorithms adopt consecutive correlation outputs to do differential coherent process and the data bit rate of BeiDou is $1 \mathrm{kbps}$ due to NH codes, these algorithms are appropriate for legacy GPS signals but not BeiDou signals.

Because of the $\mathrm{NH}$ codes, the data bit rate of BeiDou signals increases to $1 \mathrm{kbps}$ and the integration time is limited to $1 \mathrm{~ms}$. In order to extend the coherent integration time of the tracking loops, a bit synchronization algorithm is necessary to detect the position of the data bit edges and NH codes. In general, for BeiDou weak signals without frequency deviation, the maximum likelihood method can be used for bit synchronization, but considering a frequency deviation larger than $25 \mathrm{~Hz}$, a more appropriate and effective bit synchronization algorithm for BeiDou signals is necessary.

The paper proposes a bit synchronization algorithm for BeiDou weak signals with large frequency deviation. Compared with the previous research in this field, the paper has three novel contributions: (i) the differential coherent method is used to remove the effect of frequency deviation, and the differential delay time is set to be a multiple of bit cycle to remove the influence of $\mathrm{NH}$ code; (ii) the maximum likelihood function detection is used to improve the detection probability of weak signals; (iii) Monte Carlo simulations and real data tests are carried out to verify the reliability and performance improvement of the proposed algorithm.

The rest of this paper is organized as follows: Section 2 describes the BeiDou signal model and the conventional bit synchronization method. Section 3 gives detailed descriptions of the proposed algorithm. Section 4 presents the results and analysis of the Monte Carlo simulations. Finally, Section 5 is the conclusion.

\section{Signal Model and Conventional Algorithm Analysis}

\subsection{Signal Model}

Unlike GPS, the space constellation of the BeiDou System consists of Geostationary Earth Orbit (GEO) satellites, Medium Earth Orbit (MEO) satellites and Inclined Geosynchronous Satellite Orbit (IGSO) satellites [21]. The open service signal consists of B1I signal and B2I signal [22]. The signal is composed of the carrier frequency, ranging code and navigation message. The nominal frequency of the B1I signal is $1561.098 \mathrm{MHz}$, and the nominal frequency of the B2I signal is $1207.140 \mathrm{MHz}$. The chip rate of the B1I and B2I ranging code is $2.046 \mathrm{Mcps}$, and the length is 2046 chips. Navigation messages are formatted in D1 and D2 based on their rate and structure. MEO/IGSO and GEO satellites broadcast 
D1 and D2 navigation messages respectively. For D1 navigation message in format D1 of rate 50 bps, a secondary code of Neumann-Hoffman $(\mathrm{NH})$ code is modulated on ranging code. The period of the $\mathrm{NH}$ code is selected to match the duration of a navigation message bit. The bit duration of $\mathrm{NH}$ code is the same as one period of the ranging code. The duration of one navigation message bit is 20 milliseconds and the ranging code period is 1 millisecond. Thus the NH code $(-1,-1,-1,-1,-1$, $1,-1,-1,1,1,-1,1,-1,1,-1,-1,1,1,1,-1)$ with length of 20 bits, rate $1 \mathrm{kbps}$ and bit duration of 1 millisecond is adopted. It is modulated on the ranging code synchronously with navigation message bit, as shown in Figure 1. In addition, the rate of D2 navigation message is 500 bps [22].

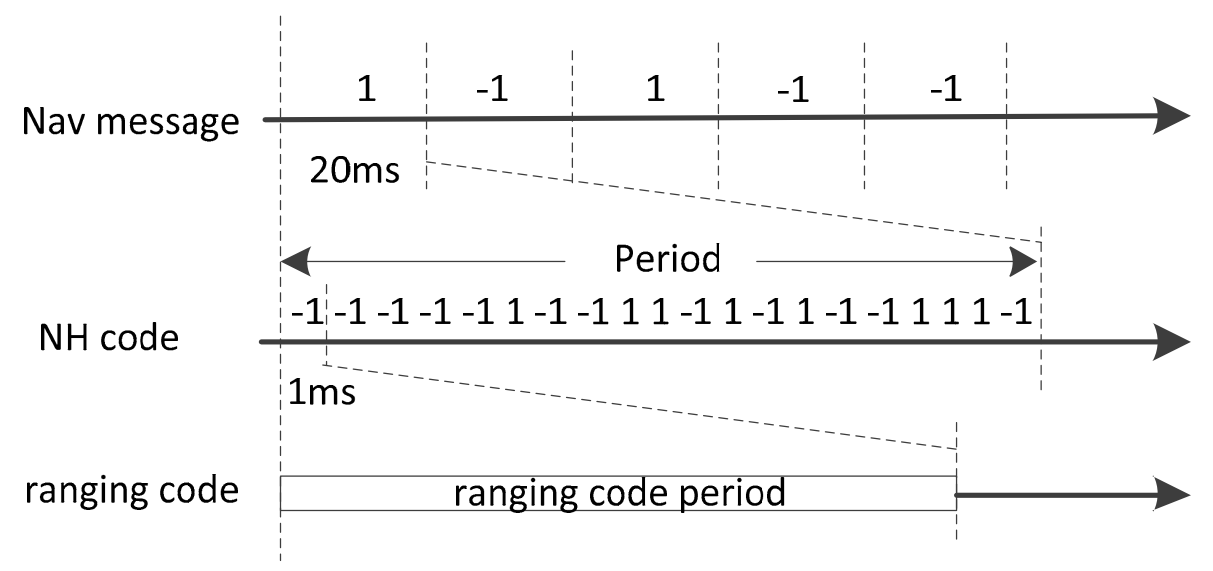

Figure 1. Signal structure of B1I and B2I signal broadcasted by MEO/IGSO and GEO satellites.

This paper mainly discusses about the B1I and B2I signal broadcast by MEO/IGSO satellites which is modulated by NH code. The primary symbols and variables used are listed in Table 1.

The signal at the input of a receiver is generally an intermediate frequency (IF) signal, obtained by down-converting, sampling and quantization. The IF signal can be written as follows [22]:

$$
s_{I F}(n)=A D\left(n T_{s}\right) H\left(n T_{s}\right) c\left(n T_{s}-\tau_{0}\right) \exp \left[j \cdot 2 \pi\left(f_{I F}+f_{d}\right) n T_{s}+\phi_{0}\right]
$$

where $A$ stands for the carrier amplitude, $D$ and $H$ represent the navigation data and the $\mathrm{NH}$ code, and $T_{s}$ is sampling time, $c(\cdot)$ represents the ranging code sequence, $\tau_{0}, \phi_{0}, f_{I F}$ and $f_{d}$ are code propagation delay, initial carrier phase, the carrier IF and Doppler shift.

After signal acquisition, the receiver obtains the approximate incoming frequency $\hat{f}_{d, L}$ and ranging code phase $\hat{\tau}_{L}$. The locally generated signal sequence is:

$$
s_{L}(n)=c\left(n T_{s}-\hat{\tau}_{L}\right) \exp \left[j \cdot 2 \pi\left(f_{I F}+\hat{f}_{d, L}\right) n T_{s}\right]
$$

Assuming the coherent integration time is $T_{c}$, the output of the kth coherent integration is [14]:

$$
Y(k)=\frac{1}{N_{c}} \sum_{n=k N_{c}}^{(k+1) N_{c}-1} s_{I F}(n) \cdot s_{L}(n)=0.5 A D_{k} H_{k} R(\delta \tau) \operatorname{sinc}\left[\delta f_{d}(k) T_{c}\right] \exp \left[j \cdot \phi_{k}+j \pi \delta f_{d}(k) T_{c}\right]
$$

where $\mathrm{k}$ denotes the index of the coherent integration interval, $N_{c}=T_{c} / T_{s}$ is the number of samples in the coherent integration time $T_{\mathcal{c}}, D_{k}= \pm 1$ is navigation data, $H_{k}= \pm 1$ is $\mathrm{NH}$ code, $R(\cdot)$ represents the autocorrelation function of the ranging code, $\delta \tau$ and $\delta f_{d}$ are the errors of code delay estimation and the Doppler shift, $\phi_{k}$ is the initial carrier phase error in the kth coherent integration interval. 
Table 1. Primary symbols and variables.

\begin{tabular}{cc}
\hline Variable & Meaning \\
\hline$s_{I F}(n)$ & received IF signal \\
$A$ & carrier amplitude \\
$D(n)$ & navigation data \\
$H(n)$ & NH code \\
$c(\cdot)$ & ranging code \\
$T_{S}$ & sampling time \\
$f_{I F}$ & carrier IF frequency \\
$f_{d}$ & Doppler frequency shift \\
$\tau_{0}$ & code propagation delay \\
$\phi_{0}$ & initial carrier phase \\
$s_{L}(n)$ & locally generated signal \\
$\hat{\tau}_{L}$ & ranging code phase of local signal \\
$\hat{f}_{d, L}$ & carrier frequency shift of local signal \\
$Y(k)$ & output of the kth coherent integration \\
$T_{c}$ & integration time \\
$N_{c}$ & the number of samples in the integration time \\
$R(\cdot)$ & autocorrelation function of the ranging code \\
$\delta \tau$ & code phase estimation error \\
$\delta f_{d}$ & Doppler frequency estimation error \\
$\phi_{k}$ & carrier phase estimation error \\
$W(k)$ & window function \\
$C\left(n, l_{b}\right)$ & cross-correlation between $Y(k)$ and $W(k)$ \\
$S\left(\hat{l}_{b}\right)$ & sum of the absolute values of cross-correlation \\
$l_{b}$ & estimate of the bit boundary \\
$T_{c o h}$ & coherent integration time \\
$Q(k)$ & differential coherent values \\
$Z(k)$ &
\end{tabular}

\subsection{Maximum Likelihood Bit Synchronization Method}

The maximum likelihood bit synchronization method uses a likelihood function to detect bit boundary locations. The likelihood function used is the sum of the absolute values of cross-correlation function between the prompt correlator output sequence and a window function. In this method, every bit transition can contribute to the detection of the bit boundary, and more bit transitions at the same bit boundary can help to improve the successful synchronization rate (SSR). The ML bit synchronization algorithm is summarized below.

Firstly, there are some parameters that need to be explained. The coherent integration time $T_{\mathcal{c}}$ in the tracking stage is $1 \mathrm{~ms}$. The ratio between data bit period and ranging code period is 20, so assume there are 20 possible bit locations. Besides, the length of data sequence to be processed in ML method is $\mathrm{N}$ bits. For GPS signals, the $20 \mathrm{~ms}$ width window function is defined as:

$$
W(k)=1,(k=1,2, \ldots 20)
$$

For BeiDou signals, the $20 \mathrm{~ms}$ width window function is the same with the $\mathrm{NH}$ code and is given by:

$$
W(k)=[-1,-1,-1,-1,-1,1,-1,-1,1,1,-1,1,-1,1,-1,-1,1,1,1,-1],(k=1,2, \ldots 20) .
$$

The cross-correlation between $Y(k)$ and $W(k)$ is given by:

$$
C\left(n, l_{b}\right)=\sum_{k=1}^{20} Y\left(20 n+l_{b}+k\right) W(k),(n=0,2, \ldots N-1),
$$


where $l_{b}$ is the initial edge shift of the window function in one bit period. The sum of the absolute values of cross-correlation is:

$$
S\left(l_{b}\right)=\sum_{n=0}^{N-1}\left|C\left(n, l_{b}\right)\right|
$$

Then the ML estimate of the bit boundary is obtained as:

$$
\hat{l}_{b}=\underset{l_{b} \in[1: 20]}{\operatorname{argmax} S} S\left(l_{b}\right)
$$

Figure 2 is the diagram of the ML bit synchronization method. The maximum sum of the absolute values of cross-correlation corresponding with the real bit boundary can be simply considered as coherent integration of $20 \mathrm{~ms}$ and non-coherent integration of $\mathrm{N}$ bits $(20 \mathrm{~N} \mathrm{~ms})$. Therefore, with longer data sequences or integration times, the performance for weak signals starts to improve.

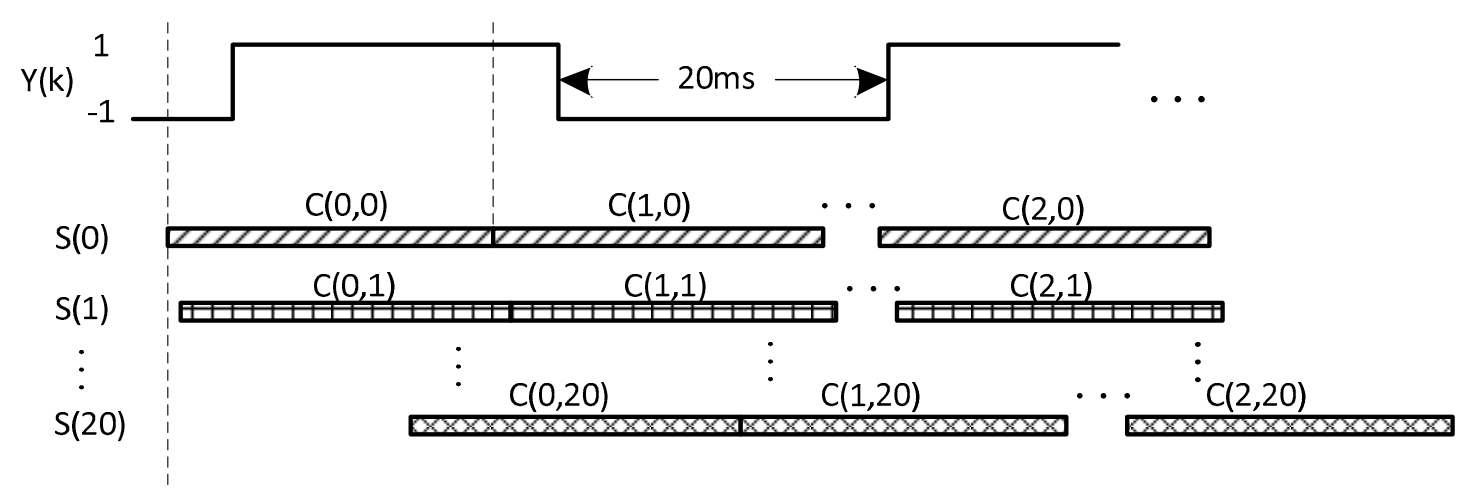

Figure 2. Diagram of the ML bit synchronization method.

\subsection{Influence Factors Analysis}

As analyzed above, the maximum sum of the absolute values of cross-correlation can be expressed as:

$$
\begin{aligned}
S\left(\hat{l}_{b}\right) & =\sum_{n=0}^{N-1}\left|C\left(n, \hat{l}_{b}\right)\right|=\sum_{n=0}^{N-1}\left|\sum_{k=1}^{20} Y\left(20 n+\hat{l}_{b}+k\right)\right| \\
& =\sum_{n=0}^{N-1}\left|0.5 A D_{k} R(\delta \tau) \operatorname{sinc}\left[\delta f_{d}(k) T_{c o h}\right] \exp \left[j \cdot \phi_{k}+j \pi \delta f_{d}(k) T_{c o h}\right]\right|
\end{aligned},
$$

where the coherent integration time $T_{\text {coh }}$ is $20 \mathrm{~ms}$.

In the tracking stage, the code phase offset is small and $R(\cdot)$ is close to the peak value, so $\operatorname{sinc}\left[\delta f_{d}(k) T_{c o h}\right]$ represents the amplitude attenuation of coherent integration due to the frequency deviation. Because the coherent integration time of tracking is $1 \mathrm{~ms}$, the frequency deviation is limited within $[-250 \mathrm{~Hz}, 250 \mathrm{~Hz}]$, but the coherent integration time $T_{\text {coh }}$ of the ML method is $20 \mathrm{~ms}$. As shown in Figure 3, the amplitude shall be attenuated to zero with a frequency deviation of an integral multiple of $50 \mathrm{~Hz}$. If the frequency deviation is $25 \mathrm{~Hz}$, the amplitude attenuation $10 \lg \operatorname{sinc}\left[\delta f_{d}(k) T_{\text {coh }}\right]$ shall be $-1.9 \mathrm{~dB}$. The results in [16] and [18] show that if the frequency deviation is larger than $25 \mathrm{~Hz}$, the ML method is nullified. 


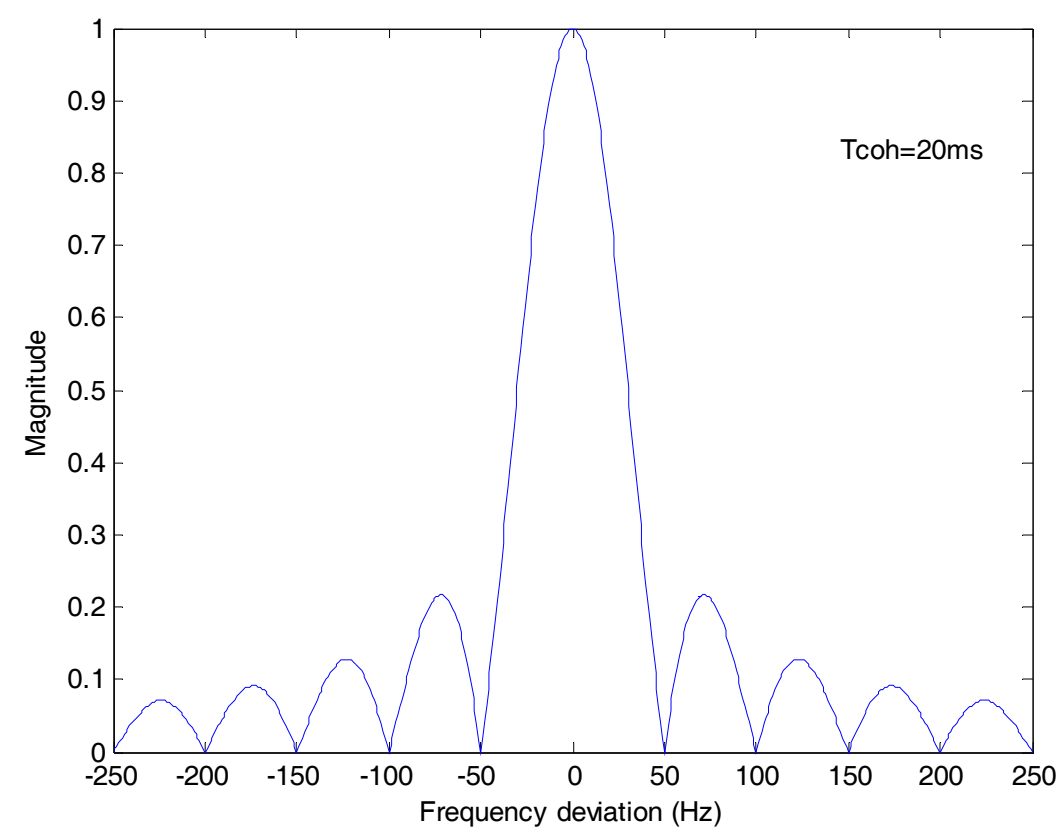

Figure 3. The amplitude attenuation of ML bit synchronization method due to frequency deviation. The coherent integration time $T_{c o h}$ is $20 \mathrm{~ms}$.

\section{Proposed Differential Coherent Bit Synchronization Algorithm}

\subsection{Differential Coherent Algorithm}

The differential coherent algorithm for bit synchronization was first introduced in [19]. The differential coherent algorithm is implemented on the coherent integration output, by multiplying the present coherent integration result with the delay conjugate of the last one. The mathematical representation of the conventional DC method is:

$$
\begin{aligned}
Q(k) & =Y(k) \cdot Y(k-1)^{*}=[0.5 A R(\delta \tau)]^{2} \exp \left(j \cdot \pi \delta f_{d} T_{c}\right) \\
& \times D_{k} D_{k-1} H_{k} H_{k-1} \operatorname{sinc}\left[\delta f_{d}(k) T_{c}\right] \operatorname{sinc}\left[\delta f_{d}(k-1) T_{c}\right]
\end{aligned}
$$

where ()$^{*}$ denotes the conjugate operation, the coherent integration time $T_{c}$ is $1 \mathrm{~ms}, \pi \delta f_{d} T_{c}$ is the carrier phase deviation between $Y(k)$ and $Y(k-1)$. The sign of $Q(k)$ changes due to bit transitions.

Assuming the integration time is $20 \mathrm{~ms}$, the accumulation output of differential coherent values is defined as:

$$
\begin{aligned}
\sum_{k=1}^{u} Q(k) & =\sum_{k=1}^{u} Y(k) \cdot Y(k-1)^{*}=[0.5 A R(\delta \tau)]^{2} \exp \left(j \cdot 2 \pi \delta f_{d} T_{c}\right) \\
& \times \sum_{k=1}^{u} D_{k} D_{k-1} H_{k} H_{k-1} \operatorname{sinc}\left[\pi \delta f_{d}(k) T_{c}\right] \operatorname{sinc}\left[\pi \delta f_{d}(k-1) T_{c}\right]
\end{aligned}
$$

where $u$ is the accumulation time.

For GPS signals, because the sign of product can only change at each bit boundary, there is at most one change in $20 \mathrm{~ms}$ and it corresponds with the real bit boundary, but for BeiDou signals, the data bit rate increases to $1 \mathrm{kbps}$ and 10 bit transitions occur in every navigation data bit, so the algorithm for BeiDou signals must be different.

\subsection{Differential Coherent Bit Synchronization Algorithm}

In order to eliminate the influence of frequency deviation, the differential coherent algorithm is combined with the maximum likelihood bit synchronization method. For BeiDou weak signals, bit 
transitions are more frequent with $\mathrm{NH}$ codes. The differential coherent process of two consecutive correlation outputs is not suitable for BeiDou signals.

According to the BeiDou signal structure and $\mathrm{NH}$ code modulation, the period of $\mathrm{NH}$ code is as long as the duration of a navigation message bit, so $H_{k}=H_{k-20 m}$, where $\mathrm{m}$ is an integer. In Equation (10), the signs of $Q(k)$ depend on $D_{k} D_{k-1} H_{k} H_{k-1}$. If the delay time of differential coherent is selected to be an integral multiple of $20 \mathrm{~ms}, H_{k} H_{k-20 m}$ will be equal to 1 and $D_{k} D_{k-20 m} H_{k} H_{k-20 m}$ will be equal to $D_{k} D_{k-20 m}$ which means that the differential coherent result will only depend on transitions of the navigation data and have nothing to do with $\mathrm{NH}$ code transitions.

Therefore, in the proposed scheme, the differential coherent delay time is modified to an integral multiple of $20 \mathrm{~ms}$ to eliminate the influence of $\mathrm{NH}$ code. The concrete steps of the proposed scheme are as follows:

- $\quad$ Step 1. Differential coherent algorithm with long delay time

The delay time of differential coherent is modified to an integral multiple of $20 \mathrm{~ms}$ and $H_{k}=H_{k-20 m}$. The mathematical representation is:

$$
\begin{aligned}
Z(k) & =Y(k) \cdot Y(k-20 m)^{*}=[0.5 A R(\delta \tau)]^{2} \exp \left(j \cdot \pi \delta f_{d} m T_{c}\right) \\
& \times D_{k} D_{k-20 m} H_{k} H_{k-20 m} \operatorname{sinc}\left[\delta f_{d}(k) T_{c}\right] \operatorname{sinc}\left[\delta f_{d}(k-20 m) T_{c}\right] \\
& =[0.5 A R(\delta \tau)]^{2} \exp \left(j \cdot \pi \delta f_{d} m T_{c}\right) \\
& \times D_{k} D_{k-20 m} \operatorname{sinc}\left[\delta f_{d}(k) T_{c}\right] \operatorname{sinc}\left[\delta f_{d}(k-20 m) T_{c}\right]
\end{aligned}
$$

where $m$ is an integral number, $\pi \delta f_{d} m T$ is the carrier phase deviation between $Y(k)$ and $Y(k-20 m)$ and it does not change with time.

Because:

$$
\left|\exp \left(j \cdot \pi \delta f_{d} m T_{c}\right)\right|=\left|\cos \left(\pi \delta f_{d} m T_{c}\right)+j \cdot \sin \left(\pi \delta f_{d} m T_{c}\right)\right|=1
$$

$\exp \left(j \cdot \pi \delta f_{d} m T_{c}\right)$ has no effect on the amplitude of $Z(k)$.

$\operatorname{sinc}\left[\delta f_{d}(k) T_{c}\right] \operatorname{sinc}\left[\delta f_{d}(k-20 m) T_{c}\right]$ represents the amplitude attenuation of the differential coherent result due to the frequency deviation. In stable tracking stage, $\delta f_{d}$ changes slightly, so $\delta f_{d}(k)$ can be approximately equal to $\delta f_{d}(k-20 m)$ and $\operatorname{sinc}\left[\delta f_{d}(k) T_{c}\right] \operatorname{sinc}\left[\delta f_{d}(k-20 m) T_{c}\right]$ can be approximately equal to $\sin c\left[\delta f_{d}(k) T_{c}\right]^{2}$. Here, $T_{c}$ is $1 \mathrm{~ms}$, and the frequency deviation is limited in $[-250 \mathrm{~Hz}, 250 \mathrm{~Hz}]$. Under the worst frequency deviation, the maximum $\operatorname{loss} 10 \lg \left\{\operatorname{sinc}\left[\delta f_{d}(k) T_{c}\right]^{2}\right\}$ is about $-0.9121 \mathrm{~dB}$.

Figure 4 is the plot of $\operatorname{sinc}\left[\delta f_{d}(k) T_{c}\right]^{2}$ which stands for the amplitude attenuation due to the frequency deviation. The frequency deviation effect is greatly eliminated compared with Figure 3.

Figure 5 shows the signs of data in the differential coherent algorithm with long delay time. It can be seen that after differential coherent process, the signs of $Z(k)$ only depend on transitions of the navigation data and have nothing to do with $\mathrm{NH}$ code.

- $\quad$ Step 2. Maximum likelihood bit synchronization method

Based on differential coherent algorithm with long delay time, the ML bit synchronization method uses the sum of the absolute values of cross-correlation function between $Z(k)$ and the window function. The 20 ms width window function is:

$$
W(k)=1,(k=1,2, \ldots 20) .
$$

The cross-correlation between $Z(k)$ and $W(k)$ is given by:

$$
C\left(n, l_{b}\right)=\sum_{k=1}^{20} Z\left(20 n+l_{b}+k\right) W(k),(n=0,2, \ldots N-1) .
$$


The sum of the absolute values of cross-correlation is:

$$
S\left(l_{b}\right)=\sum_{n=0}^{N-1}\left|C\left(n, l_{b}\right)\right| .
$$

Then the ML estimate of bit boundaries is obtained as:

$$
\hat{l}_{b}=\underset{l_{b} \in[1: 20]}{\operatorname{argmax}} S\left(l_{b}\right) .
$$

The maximum sum of the absolute values of cross-correlation can be expressed as:

$$
\begin{aligned}
S\left(\hat{l}_{b}\right) & =\sum_{n=0}^{N-1}\left|C\left(n, \hat{l}_{b}\right)\right|=\sum_{n=0}^{N-1}\left|\sum_{k=1}^{20} Z\left(20 n+\hat{l}_{b}+k\right)\right| \\
& =\sum_{n=0}^{N-1}\left|[0.5 A R(\delta \tau)]^{2} \exp \left(j \cdot \pi \delta f_{d} m T_{c}\right) \sum_{k=1}^{20} D_{k} D_{k-20 m} \operatorname{sinc}\left[\delta f_{d}(k) T_{c}\right] \operatorname{sinc}\left[\delta f_{d}(k-20 m) T_{c}\right]\right|
\end{aligned},
$$

here, $\sum_{k=1}^{20} \sin c\left[\delta f_{d}(k) T_{c}\right] \sin c\left[\delta f_{d}(k-20 m) T_{c}\right]$ represents the frequency deviation effect of the proposed algorithm and $\sin c\left[\delta f_{d}(k) T_{c o h}\right]$ in Equation (9) represents the frequency deviation effect of the conventional ML algorithm. In order to compare the frequency deviation effects of the two algorithms, we should make approximations to $\sum_{k=1}^{20} \sin c\left[\delta f_{d}(k) T_{c}\right] \sin c\left[\delta f_{d}(k-20 m) T_{c}\right]$. Because $\delta f_{d}$ changes slightly in stable tracking stage, $\delta f_{d}(k)$ can be approximately equal to $\delta f_{d}(k-20 m)$ and $\sin c\left[\delta f_{d}(k) T_{c}\right] \sin c\left[\delta f_{d}(k-20 m) T_{c}\right]$ can be approximately equal to $\sin c\left[\delta f_{d}(k) T_{c}\right]^{2}$ as:

$$
\sum_{k=1}^{20} \operatorname{sinc}\left[\delta f_{d}(k) T_{c}\right] \operatorname{sinc}\left[\delta f_{d}(k-20 m) T_{c}\right] \approx \sum_{k=1}^{20}\left\{\operatorname{sinc}\left[\delta f_{d}(k) T_{c}\right]\right\}^{2} \approx 20\left\{\operatorname{sinc}\left[\delta f_{d}(k) T_{c}\right]\right\}^{2}
$$

In Figure 6, the red line stands for $10 \times \lg \left(20\left\{\operatorname{sinc}\left[\delta f_{d}(k) T_{c}\right]\right\}^{2}\right)$ and the blue line stands for $10 \times \lg \left(\operatorname{sinc}\left[\delta f_{d}(k) T_{c o h}\right]\right)$. It can be seen that the absolute value of the former is much smaller than the latter especially with a frequency deviation of an integral multiple of $50 \mathrm{~Hz}$, because $T_{c}$ is $1 \mathrm{~ms}$ and $T_{\text {coh }}$ is $20 \mathrm{~ms}$.

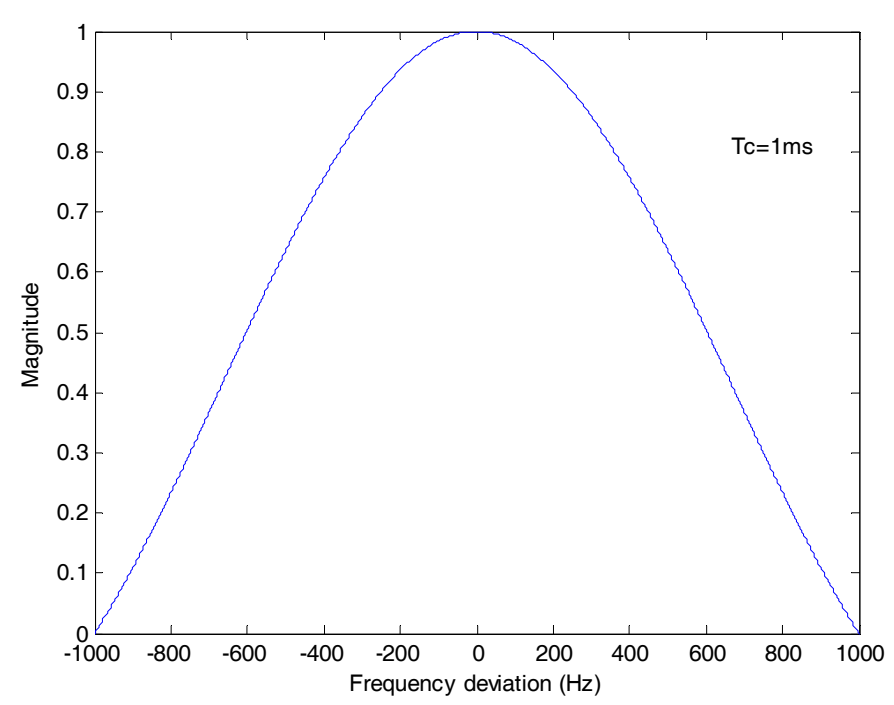

Figure 4. The amplitude attenuation of differential coherent algorithm due to frequency deviation. The integration time $T_{\mathcal{c}}$ is $1 \mathrm{~ms}$. 


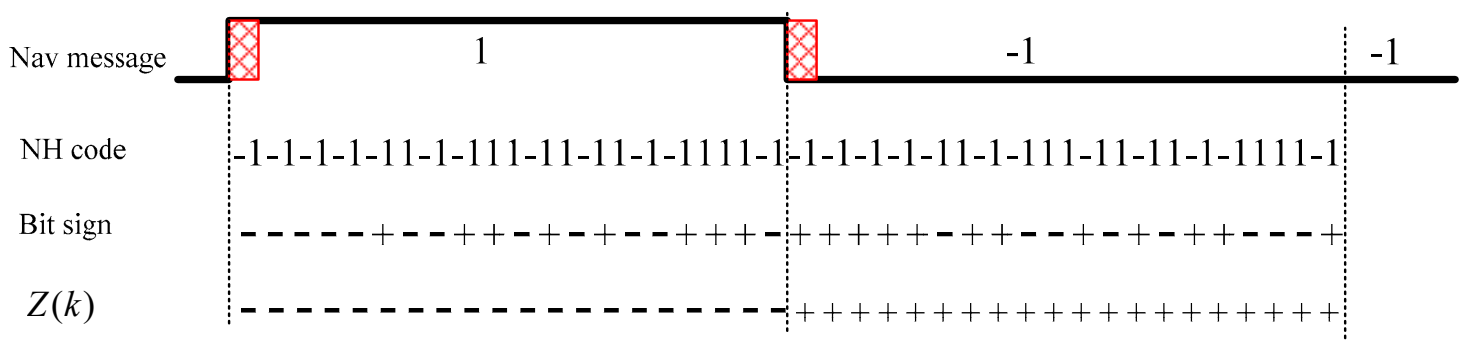

Figure 5. Data signs differential coherent algorithm with long delay time.

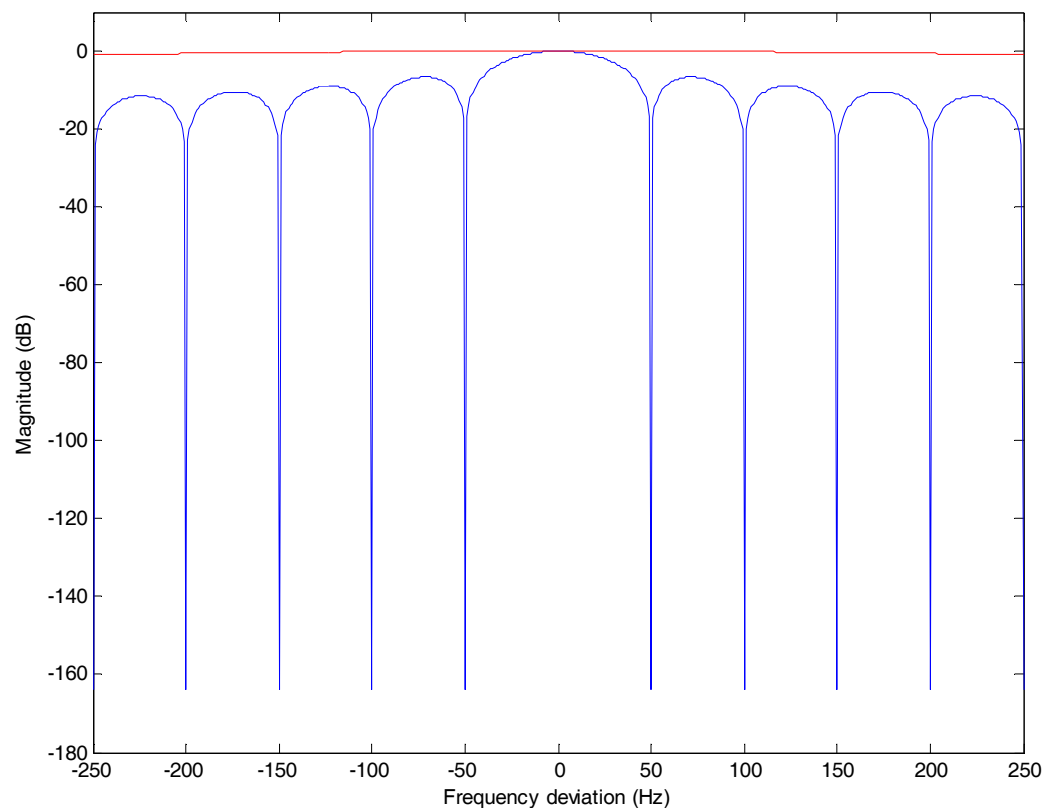

Figure 6. The amplitude attenuations of ML and DCML bit synchronization algorithms due to frequency deviation. The blue line is for ML and the red line is for DCML.

Therefore, $\sum_{k=1}^{20} \operatorname{sinc}\left[\delta f_{d}(k) T_{c}\right] \operatorname{sinc}\left[\delta f_{d}(k-20 m) T_{c}\right]$ in the Equation (19) is much smaller than $\operatorname{sinc}\left[\delta f_{d}(k) T_{c o h}\right]$ in the Equation (9). Therefore, the proposed bit synchronization algorithm should outperform the conventional ML algorithm with a frequency deviation of an integral multiple of $50 \mathrm{~Hz}$. As above, the proposed bit synchronization algorithm eliminates the influence of frequency deviation by adopting differential coherent algorithm and eliminates the influence of $\mathrm{NH}$ code by modifying the differential delay time to an integral multiple of $20 \mathrm{~ms}$.

\section{Simulation Results and Analysis}

\subsection{Monte Carlo Simulations}

To achieve a comprehensive assessment of the proposed bit synchronization scheme, Monte Carlo simulations are carried out. The simulation platform is shown in Figure 7, which consists of BeiDou B1I IF signal generation and software receiver baseband process. The parameters used in Monte Carlo simulations are provided in Table 2.

The concrete steps of the BeiDou B1I IF signal generation simulation are as follows.

(1) Position and velocity of satellites are resolved based on stored ephemeris parameters.

(2) The time delay variable is calculated according to the position and velocity of satellites and the trace. 
(3) On the basis of BeiDou signal structure, carrier, ranging code and NH code are generated.

(4) The BeiDou IF signal is generated according to the time delay variable and signal modulation.

(5) Additional white Gaussian noise is generated according to the CN0 settings.

(6) Every sampled point of the signal is quantized and stored in two bits.

The signal spectrum distribution of the IF samples is shown in Figure 8.

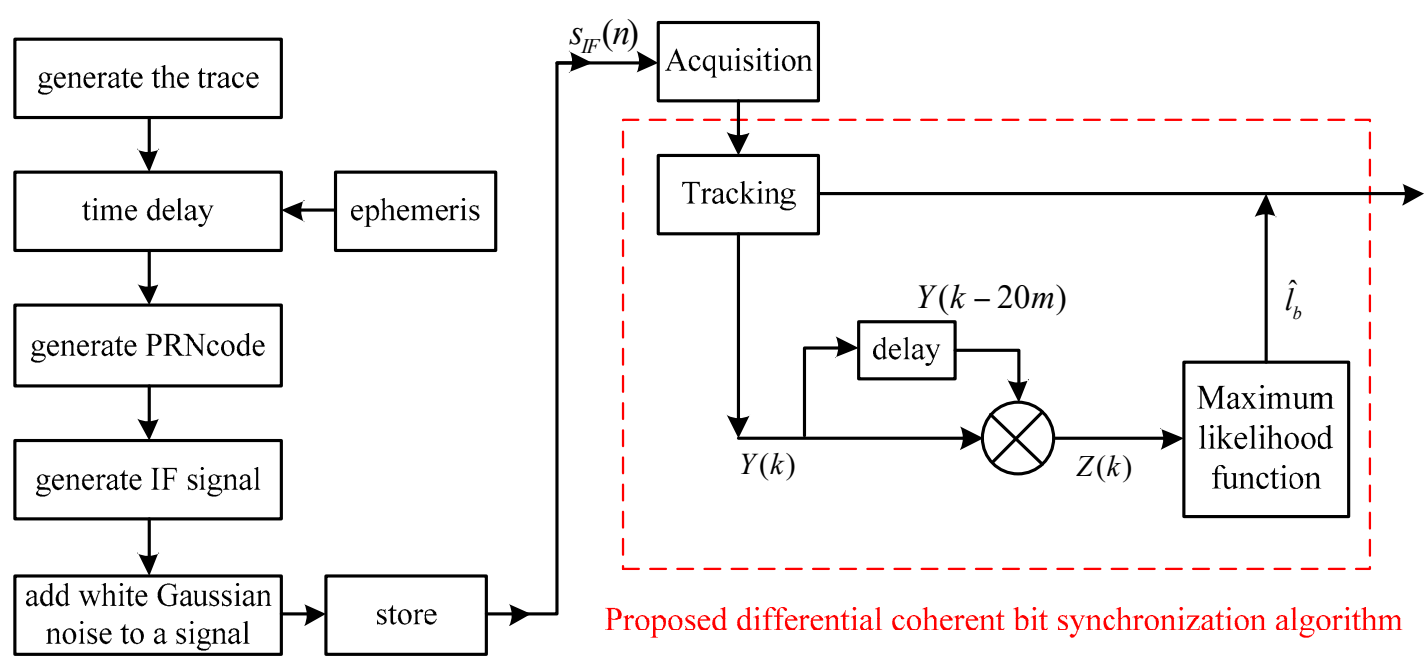

Figure 7. The diagram of the platform for Monte Carlo simulations.

Table 2. Parameters used in the Monte Carlo simulations.

\begin{tabular}{ccc}
\hline Sections & Parameters & Values \\
\hline \multirow{3}{*}{ Signal Generation } & IF frequency & $4.1304 \mathrm{MHz}$ \\
& sampling frequency & $16.3676 \mathrm{MHz}$ \\
& visible satellites & $6,7,11 \mathrm{and} \mathrm{12}$ \\
& CN0 & $20 \sim 40 \mathrm{~dB}-\mathrm{Hz}$ \\
\hline \multirow{2}{*}{ Software Receiver } & DLL bandwidth & $2 \mathrm{~Hz}$ \\
& PLL bandwidth & $40 \mathrm{~Hz}$ \\
& frequency deviation & $0,25,50 \mathrm{~Hz}$ \\
& differential coherent delay time & $20 \mathrm{~ms}$ \\
& differential coherent integration time & $40 \mathrm{~ms}$ \\
\hline
\end{tabular}

In the software receiver section, the proposed differential coherent bit synchronization algorithm is after conventional signal acquisition and tracking. Repeated acquisition based on 1 2 ms of coherent integration is used for signal acquisition, and DLL and PLL are used for signal tracking.

To prove the reliability of the proposed algorithm, tests are done to evaluate the sensitivity of different CNOs. Using Monte Carlo simulations, additional white Gaussian noise is generated for each trial, and 1000 trials are used for each probability. Monte Carlo simulations of different algorithms are carried out for comprehensive evaluation.

The first simulation tests the effect of frequency deviation on the conventional maximum likelihood bit synchronization algorithm. The detection probability is plotted as a function of the CNOs in Figure 9. Without frequency deviation, the conventional ML bit synchronization algorithm has good performance in weak signal environment. The detection probability is 0.92 at CN0 of $23 \mathrm{~dB}-\mathrm{Hz}$. However, with the increase of frequency deviation, the detection probability attenuates seriously. When the frequency deviation is $50 \mathrm{~Hz}$, the ML method is nullified. 


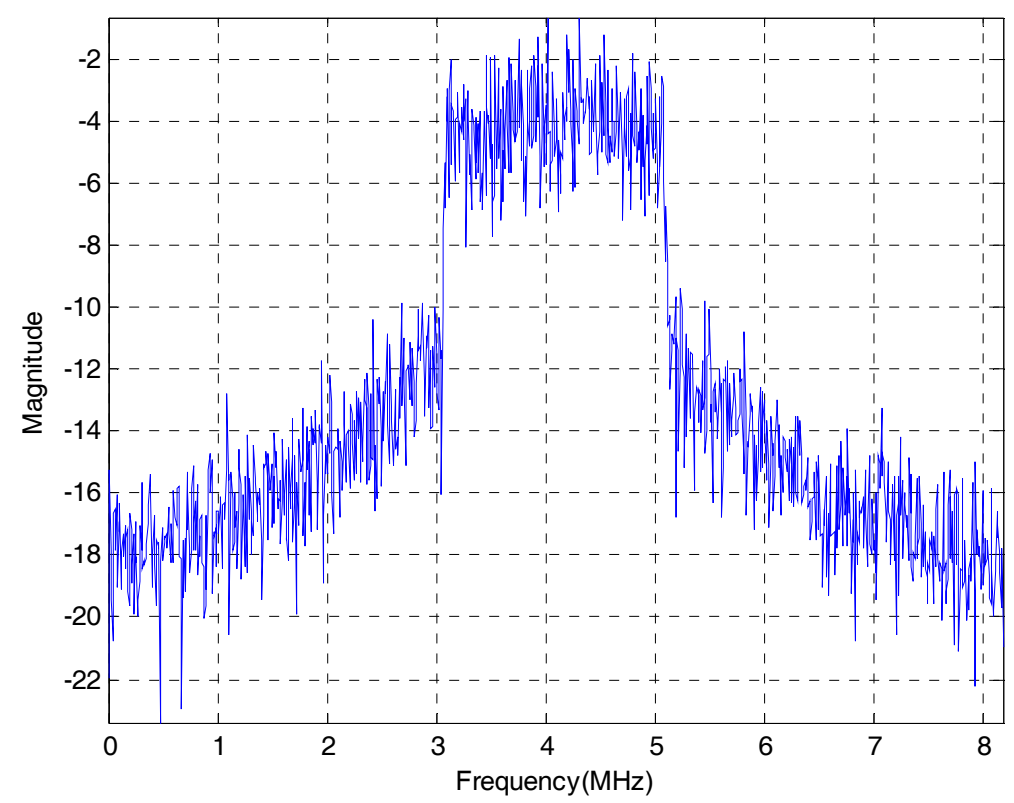

Figure 8. Signal spectrum distribution of the BeiDou B1I IF signals.

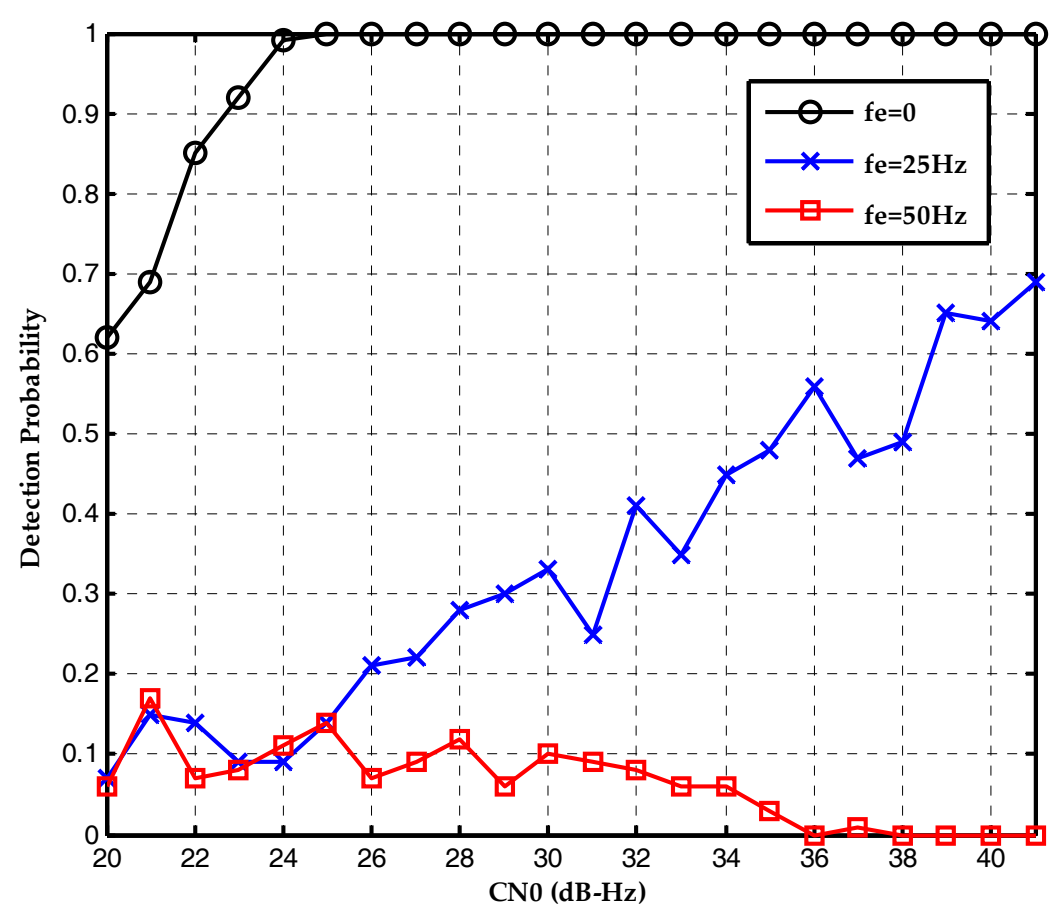

Figure 9. Detection probabilities of the ML bit synchronization algorithm under the CN0s of 20 40 dB-Hz and different frequency deviations.

Therefore, $\sum_{k=1}^{20} \operatorname{sinc}\left[\delta f_{d}(k) T_{c}\right] \operatorname{sinc}\left[\delta f_{d}(k-20 m) T_{c}\right]$ in the Equation (19) is much smaller than $\operatorname{sinc}\left[\delta f_{d}(k) T_{c o h}\right]$ in the Equation (9). Therefore, the proposed bit synchronization algorithm should outperform the conventional ML algorithm with a frequency deviation of an integral multiple of $50 \mathrm{~Hz}$. The second simulation evaluates the effect of frequency deviation on the proposed bit synchronization algorithm. The detection probability is plotted as a function of the CNOs in Figure 10. With the increase of frequency deviation, the detection probability attenuates too, but not seriously. When the frequency deviation is $50 \mathrm{~Hz}$, the detection probability is 0.72 at CN0 of $36 \mathrm{~dB}-\mathrm{Hz}$. 


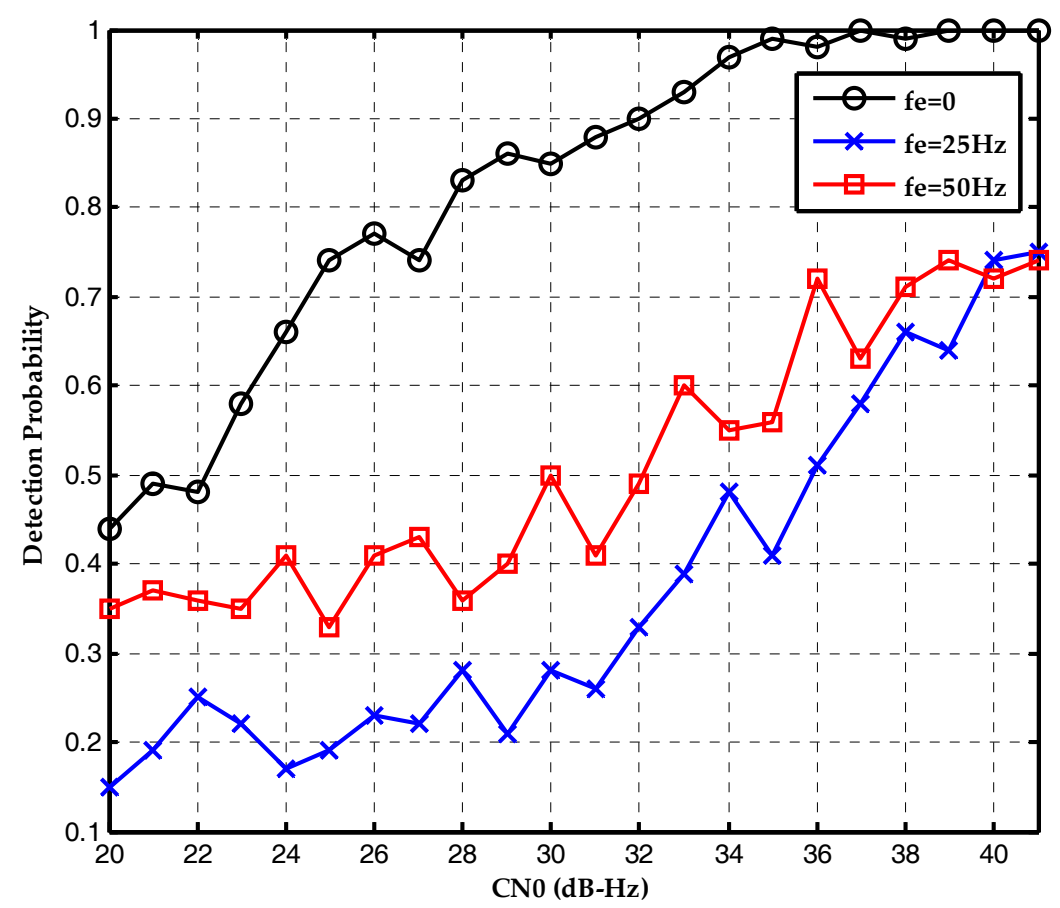

Figure 10. Detection probabilities of the DCML bit synchronization algorithm under the CN0s of 20 40 dB-Hz and different frequency deviations.

Finally, the performances of two algorithms are compared with different frequency deviations. Figure 11 is the detection probability without frequency deviation. The conventional maximum likelihood method outperforms the proposed bit synchronization algorithm which has gain attenuation. Figure 12 is the detection probability with a frequency deviation of $25 \mathrm{~Hz}$ and the performances of two algorithms are about the same. Then, Figure 13 is the detection probability with a frequency deviation of $50 \mathrm{~Hz}$. It is clear that the proposed bit synchronization algorithm outperforms the conventional maximum likelihood method and the detection probability of the proposed bit synchronization algorithm is always higher than the conventional maximum likelihood method.

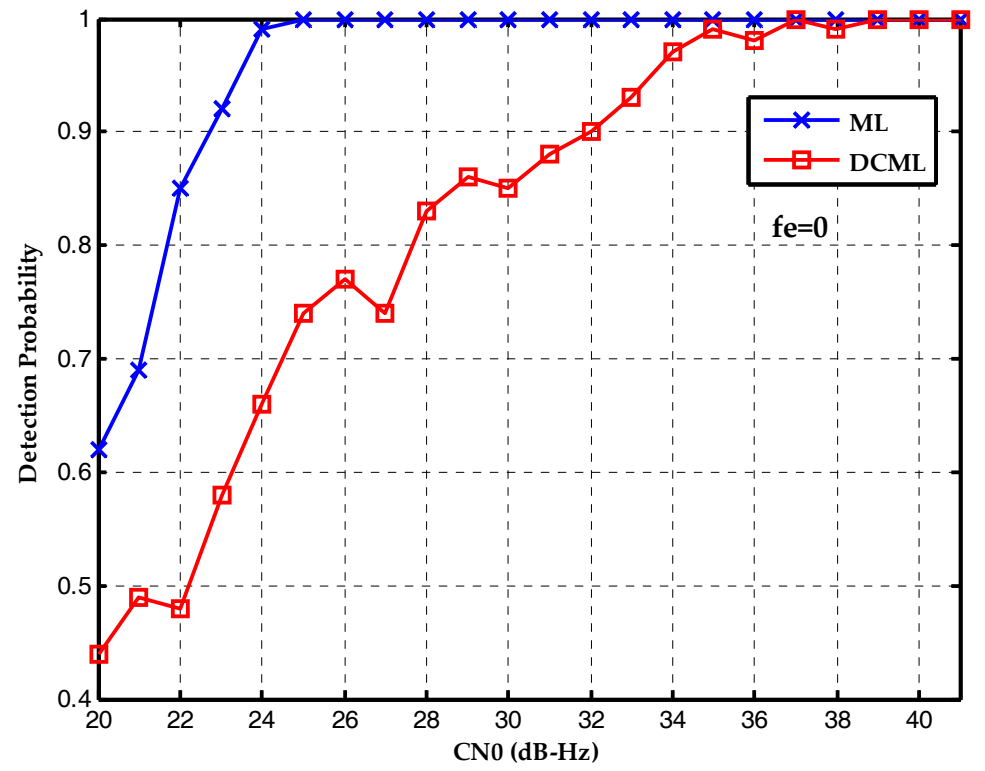

Figure 11. Detection probabilities of ML and DCML bit synchronization algorithms under the CN0s of 20 40 dB-Hz with a frequency deviation of $0 \mathrm{~Hz}$. 


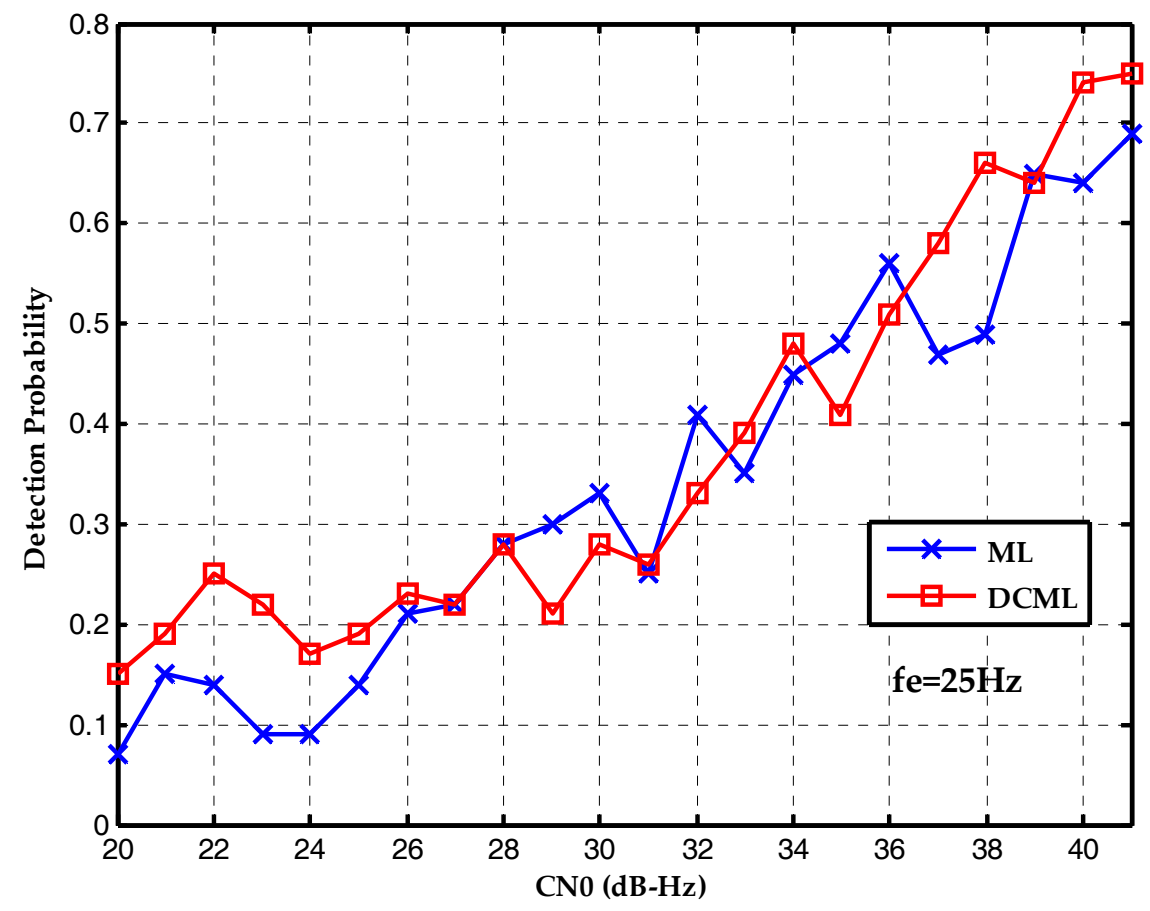

Figure 12. Detection probabilities of ML and DCML bit synchronization algorithms under the CN0s of 20 40 dB-Hz with a frequency deviation of $25 \mathrm{~Hz}$.

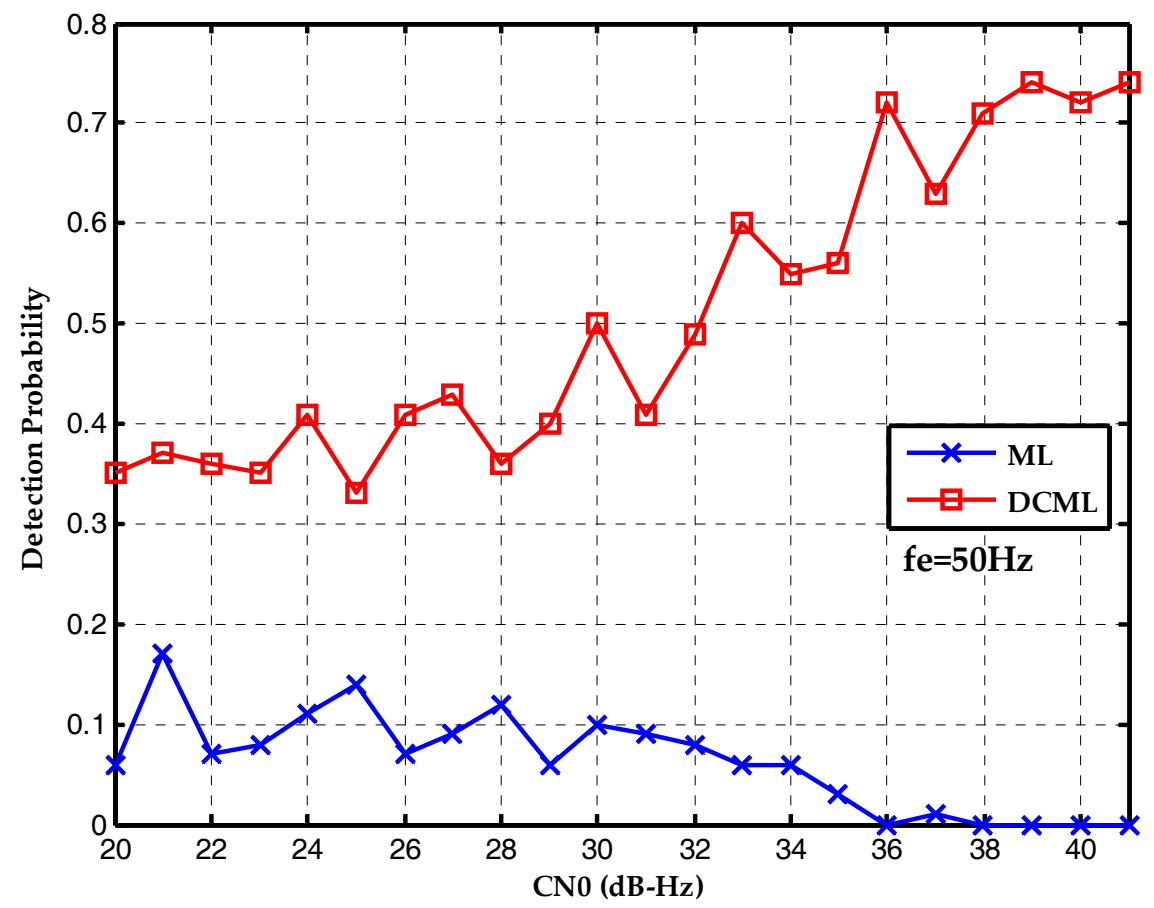

Figure 13. Detection probabilities of ML and DCML bit synchronization algorithms under the CN0s of 20 40 dB-Hz with a frequency deviation of $50 \mathrm{~Hz}$.

The results above show that the detection probability of the traditional maximum likelihood method attenuates seriously with the increase of frequency deviation and the ML method is even nullified when the frequency deviation is $50 \mathrm{~Hz}$. By contrast, the detection probability of the proposed bit synchronization algorithm does not attenuate very seriously with the increase of frequency deviation. The detection probability of the proposed bit synchronization algorithm is always higher 
than the conventional maximum likelihood method when the frequency deviation is $50 \mathrm{~Hz}$. Therefore, the proposed bit synchronization algorithm outperforms the conventional maximum likelihood method with large frequency deviation which is consistent with the theory analysis in Section 3.2.

\subsection{Real Data Tests}

To confirm the feasibility of the proposed algorithm, real data tests are conducted. The BeiDou B1I receiver used was developed by the Navigation Research Center, Nanjing University of Aeronautics and Astronautics (NRC, NUAA) and the hardware structure was developed by Shanghai Yuzhi. As shown in Figure 14, the receiver test platform consists of a DSP-TMS320C6713B and an FPGA-EP4CE115F23, and an AD8347 is used as the quadrature down-conversion mixer. The parameters used are provided in Table 3. The antenna is placed on the roof of College of Automation Engineering building of NUAA and the signal is processed in real time.

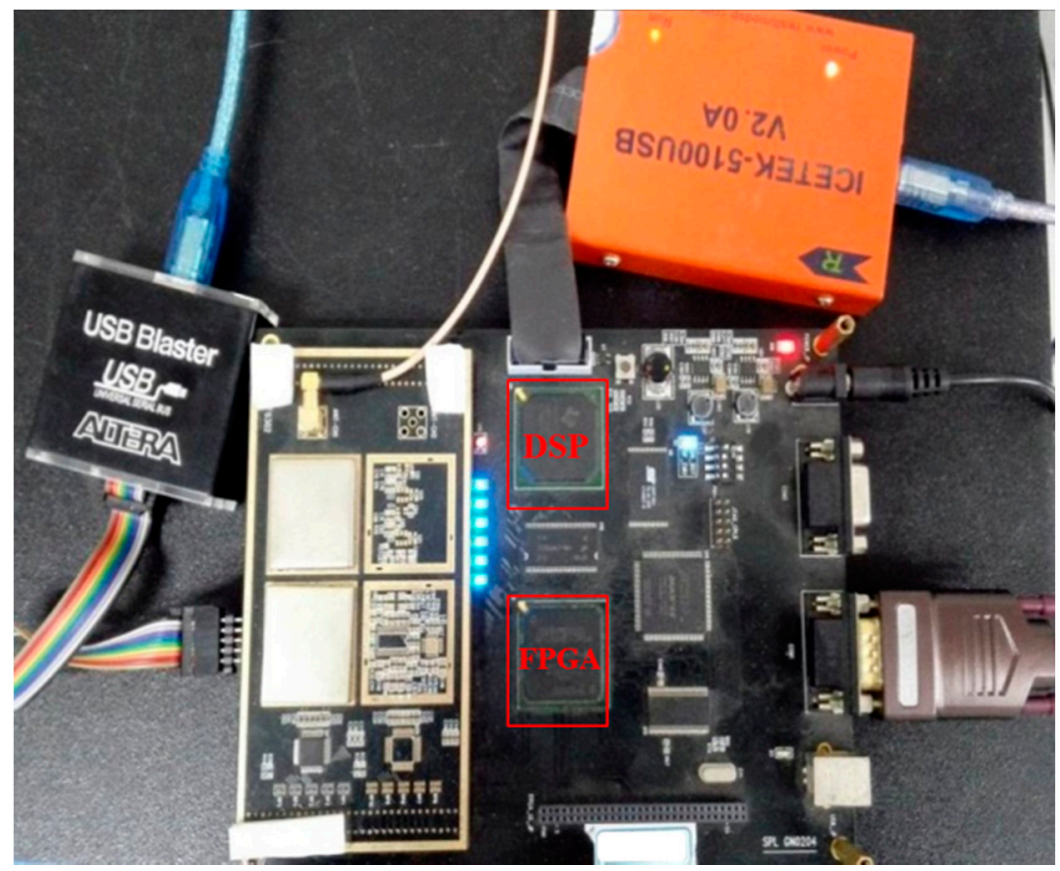

Figure 14. The BeiDou B1I receiver test platform.

Table 3. Parameters used in real data tests.

\begin{tabular}{cc}
\hline Parameters & Values \\
\hline IF frequency & $4.098 \mathrm{MHz}$ \\
Sampling frequency & $62 \mathrm{MHz}$ \\
DLL bandwidth & $5 \mathrm{~Hz}$ \\
PLL bandwidth & $20 \mathrm{~Hz}$ \\
FLL bandwith & $40 \mathrm{~Hz}$ \\
Differential coherent delay time & $20 \mathrm{~ms}$ \\
Differential coherent integration time & $40 \mathrm{~ms}$ \\
\hline
\end{tabular}

In the BeiDou B1I receiver, repeated acquisition based on 1 2 $\mathrm{ms}$ of coherent integration is used for signal acquisition. DLL and PLL assisted by FLL are used for signal tracking. The proposed differential coherent bit synchronization algorithm is processed after signal acquisition and tracking.

The number of visible satellites is ten, including five GEO, three IGSO and two MEO. Take satellite $\# 9$ as an example and the result of tracking loop is shown in Figure 15. 


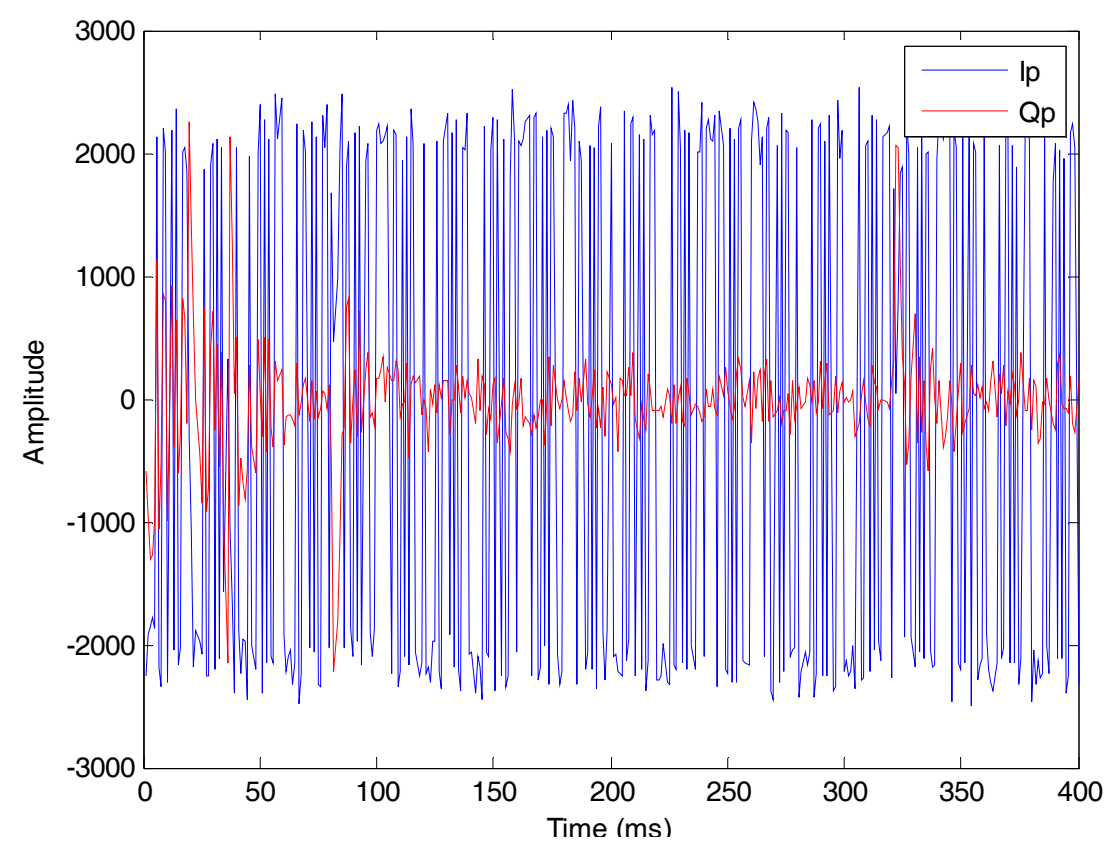

Figure 15. The tracking result of satellite \#9.

The proposed bit synchronization algorithm is processed based on the coherent integration results of tracking loops. Figure 16 is the result of differential coherent algorithm with a delay time of $20 \mathrm{~ms}$ and the signs are consistent with transitions of the navigation data. The amplitudes of DCML algorithm at all possible bit boundaries are shown in Figure 17 and the bit boundary estimation is one. After bit synchronization, the bits are decoded and position calculation is realized.

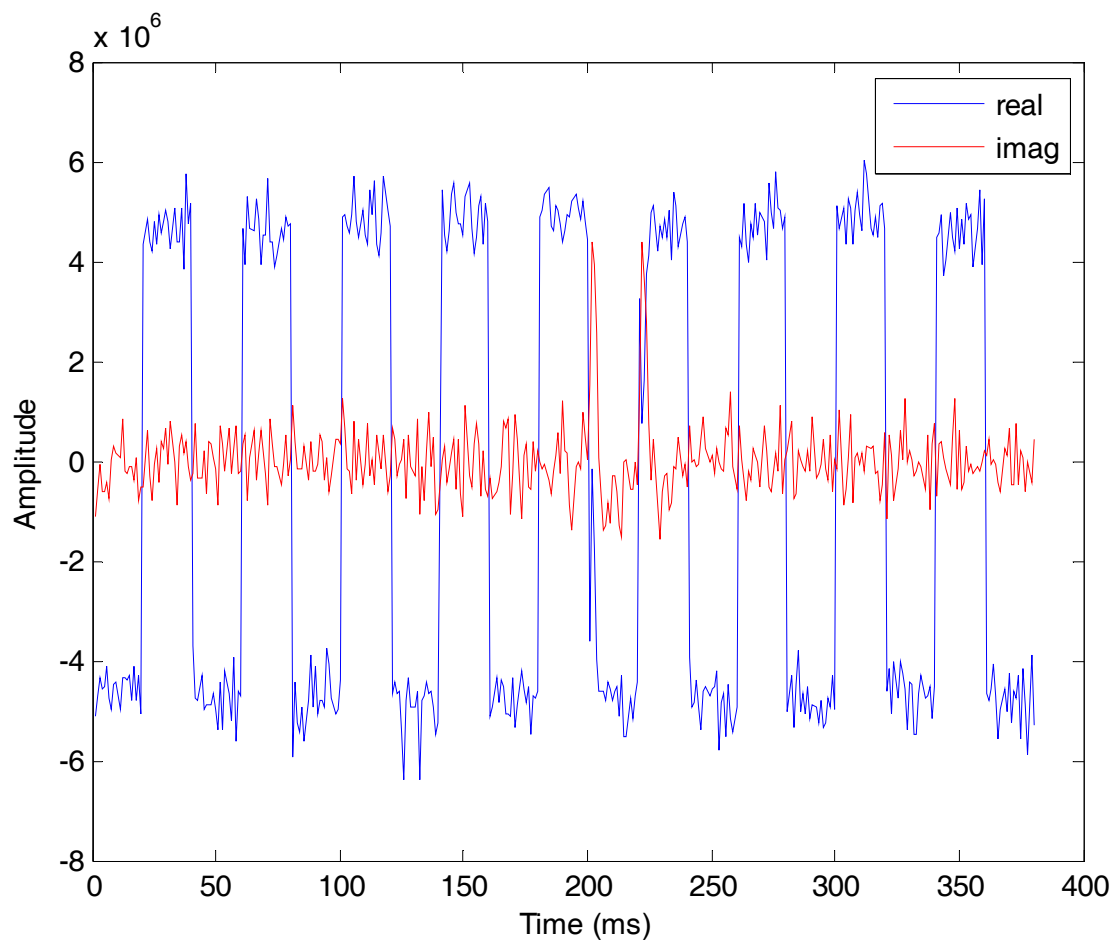

Figure 16. The differential coherent result of satellite \#9. 


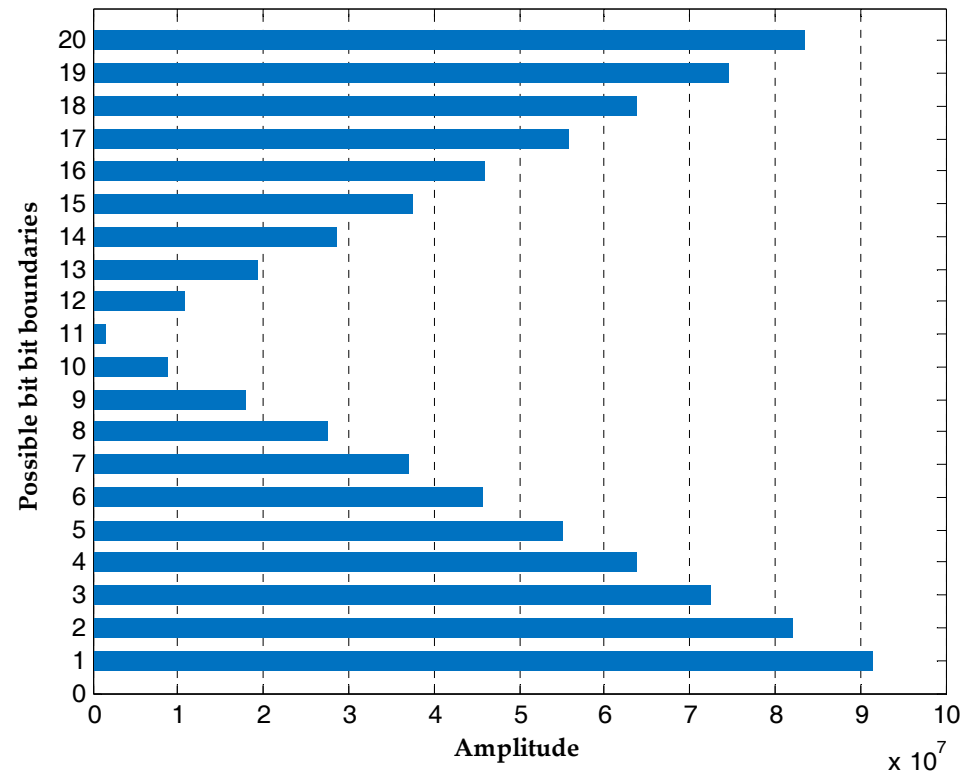

Figure 17. The amplitudes of DCML algorithm at all possible bit boundaries.

\section{Conclusions}

In order to realize bit synchronization for BeiDou weak signals with large frequency deviation, a bit synchronization algorithm based on differential correlation and maximum likelihood is proposed. Firstly, the differential correlation is used to remove the effect of frequency deviation, and the differential delay time is set to be a multiple of bit cycle to remove the influence of $\mathrm{NH}$ code. Secondly, the maximum likelihood function detection is used to improve the detection probability of weak signals.

Monte Carlo simulations are carried out to analyze the detection performance of the proposed algorithm compared with a traditional algorithm under the CN0s of $20 \sim 40 \mathrm{~dB}-\mathrm{Hz}$ and different frequency deviations. The results show that the proposed algorithm outperforms the traditional method when the frequency deviation is $50 \mathrm{~Hz}$. This algorithm can remove the effect of BeiDou NH code effectively and weaken the influence of frequency deviation. This algorithm is suitable for BeiDou weak signal bit synchronization with large frequency deviation. In addition, this method is applicable to BeiDou signals and it is also applicable to GPS, Galileo and other satellites navigation systems.

Acknowledgments: This work was partially supported by the National Natural Science Foundation of China (Grant No. 61374115, 61328301), the Funding for Outstanding Doctoral Dissertation in NUAA (Grant No. BCXJ14-06), the Funding of Jiangsu Innovation Program for Graduate Education (Grant No. KYLX_0283) "the Fundamental Research Funds for the Central Universities". The author would like to thank the anonymous reviewers for helpful comments and valuable remarks.

Author Contributions: Zhifeng Han conceived the idea and wrote the paper; Yi Wang performed the experiments; Qinghua Zeng analyzed the data; Jianye Liu and Rongbing Li coordinated the research.

Conflicts of Interest: The authors declare no conflict of interest.

\section{References}

1. Zhao, L.; Liu, A.; Ding, J.; Wang, J. BeiDou Signal Acquisition with Neumann-Hoffman Code Modulation in a Degraded Channel. Sensors 2017, 17, 323. [CrossRef] [PubMed]

2. Yan, K.; Ziedan, N.I.; Zhang, H.; Guo, W.; Niu, X.; Liu, J. Weak GPS signal tracking using FFT discriminator in open loop receiver. GPS Solut. 2016, 20, 225-237. [CrossRef]

3. Xie, P.; Petovello, M.G. Improved Correlator Peak Selection for GNSS Receivers in Urban Canyons. J. Navig. 2015, 68, 869-886. [CrossRef] 
4. Wang, X.; Ji, X.; Feng, S.; Calmettes, V. A high-sensitivity GPS receiver carrier-tracking loop design for high-dynamic applications. GPS Solut. 2015, 19, 225-236. [CrossRef]

5. Wang, X.; Ji, X.; Feng, S. A scheme for weak GPS signal acquisition aided by SINS information. GPS Solut. 2014, 18, 243-252. [CrossRef]

6. Wang, L.; Groves, P.D.; Ziebart, M.K. Smartphone shadow matching for better cross-street GNSS positioning in urban environments. J. Navig. 2015, 68, 411-433. [CrossRef]

7. Trajkovski, K.K.; Sterle, O.; Stopar, B. Sturdy positioning with high sensitivity GPS sensors under adverse conditions. Sensors 2010, 10, 8332-8347. [CrossRef] [PubMed]

8. Shanmugam, S.K. New Enhanced Sensitivity Detection Techniques for GPS L 1 C/A and Modernized Signal Acquisition. Ph.D. Thesis, Departement of Geomatics Engineering, The University of Calgary, Calgary, AB, Canada, 2008.

9. Gernot, C. Development of Combined GPS L1/L2C Acquisition and Tracking Methods for Weak Signals Environments. Ph.D. Thesis, Departement of Geomatics Engineering, The University of Calgary, Calgary, AB, Canada, 2011.

10. Zhang, P.; Xu, C.; Cai, X.; Li, H. Performance analysis of BDS for regional services with consideration on weighted factors. Proc. Inst. Mech. Eng. Part G 2016, 230, 146-156. [CrossRef]

11. Zou, D.; Deng, Z.; Huang, J.; Liu, H.; Yang, L. A study of Neuman Hoffman Codes for GNSS Application. In Proceedings of the 2009 WiCom'09 5th International Conference on Wireless Communications, Networking and Mobile Computing, Beijing, China, 24-26 September 2009; pp. 1-4.

12. Bhuiyan, M.Z.H.; Söderholm, S.; Thombre, S.; Ruotsalainen, L.; Kuusniemi, H. Overcoming the challenges of BeiDou receiver implementation. Sensors 2014, 14, 22082-22098. [CrossRef] [PubMed]

13. Ward, P.W.; Betz, J.W.; Hegarty, C.J. Satellite signal acquisition, tracking, and data demodulation. Underst. GPS Princ. Appl. 2006, 5, 174-175.

14. Parkinson, B.; Spilker, J.J.; Axelrad, P.; Enge, P. GPS: Theory and Applications 1996; Volumes 1 and 2, AIAA: Washington, DC, USA, 1996.

15. Ziedan, N.I.; Garrison, J.L. Bit Synchronization and Doppler Frequency Removal at Very Low Carrier to Noise Ratio Using a Combination of the Viterbi Algorithm with an Extended Kalman Filter. In Proceedings of the 16th International Technical Meeting of the Satellite Division of The Institute of Navigation (ION GPS/GNSS 2003), Portland, OR, USA, 9-12 September 2003; pp. 616-627.

16. Ren, T.; Petovello, M. An analysis of maximum likelihood estimation method for bit synchronization and decoding of GPS L1 C/A signals. EURASIP J. Adv. Signal Proc. 2014, 2014, 1-12. [CrossRef]

17. Puga, G.L.; Roncagliolo, P.A.; Garcia, J.G. Multi-Millisecond GNSS Maximum Likelihood Bit Synchronization Method. In Proceedings of the Fifth International Conference on Advances in Satellite and Space Communications, Venice, Italy, 22-27 April 2013; pp. 34-39.

18. Kokkonen, M.; Pietila, S. A New Bit Synchronization Method for a GPS Receiver. In Proceedings of the 2002 IEEE Position Location and Navigation Symposium, Palms Springs, CA, USA, 15-18 April 2002; pp. 85-90.

19. Li, X.; Guo, W. Efficient differential coherent accumulation algorithm for weak GPS signal bit synchronization. IEEE Commun. Lett. 2013, 17, 936-939.

20. Dengyun, L.E.I.; Weijun, L.U.; Zhang, Y.; Dunshan, Y.U. Balance Differential Coherent Bit Synchronization Algorithm for GNSS Receiver. IEICE Trans. Commun. 2015, 98, 1133-1140.

21. Jan, S.S.; Tao, A.L. Comprehensive comparisons of satellite data, signals, and measurements between the BeiDou navigation satellite system and the global positioning system. Sensors 2016, 16, 689. [CrossRef] [PubMed]

22. China Satellite Navigation Office. BeiDou Navigation Satellite System Signal in Space Interface Control Document Open Service Signal (Version 2.1). Available online: http://en.beidou.gov.cn/index.html (accessed on 18 November 2016).

(C) 2017 by the authors. Licensee MDPI, Basel, Switzerland. This article is an open access article distributed under the terms and conditions of the Creative Commons Attribution (CC BY) license (http:/ / creativecommons.org/licenses/by/4.0/). 